\title{
Colour, Texture, and Shape Features based Object Recognition Using Distance Measures
}

\author{
S.M. Mohidul Islam \\ Computer Science and Engineering Discipline, Khulna University, Khulna, Bangladesh \\ E-mail: mohid@cse.ku.ac.bd \\ Farhana Tazmim Pinki \\ Computer Science and Engineering Discipline, Khulna University, Khulna, Bangladesh \\ E-mail: farhana.kucse@gmail.com
}

Received: 22 June 2021; Accepted: 25 July 2021; Published: 08 August 2021

\begin{abstract}
Object recognition is the recognizing process of objects into semantically expressive classes using its visual insides. Classification of objects becomes complex and challenging task because of its size, poor image quality, occlusion, scaling, geometric distortion, lightening, etc. In this paper, global descriptors that means Color, Texture, and Shape features are used to recognize object. Color histogram is used to obtain the color content, texture content is obtained using Gabor wavelet, and shape content is extracted using Hough transform. These low level or global features are used for creating feature vector. Distance measure is used to find the 1-Nearest Neighbor from the training images i.e. object with minimum distance or maximum similarity with visual contents of the query image. The class of that training image is the predicted label of the query image. We have used twelve different distance measures: some are metrics, some are non-metrics and finally, their recognition accuracy is compared. Ensemble of these distance measures is also used for object recognition in the image. We evaluate this method on a publicly available object-recognition dataset: Columbia Object Image Library (COIL-100) dataset. The experiments show that the recognized results outperform many state-of-the-art methods.
\end{abstract}

Index Terms: Color Histogram, Gabor Wavelet, Hough Transform, Nearest Neighbor, Ensemble of Distance Measures.

\section{Introduction}

Object detection and classification from digital image or video is a never-ending development area which has wide-ranging applications to each user and saves time in various fields such as computer vision. A recognition technique is used for organization or disunion of various types of objects separately into the classes. There are some problems in the process of detecting target object which is hidden in most of the times because of other object presence [2]. It is crucial to detect and recognize object especially when object in images has standpoint, brightness, positioning, scale variations and obscured by other objects. Moreover, object recognition becomes a complex process when the object in image is infected by noise and gets defected or distorted [18]. For man-made object detection, it is difficult to segment the object from the background [17]. Moreover, some multi-object tracking applications, such as Digilog book, deal with many objects of different classes [21]. Humans can distinguish a mass of objects in images with petite effort, despite the fact of above described crucial situations. But in general, this is still a challenge for machine based systems [22]. The objects of image can be predicted accurately based on their visual geographies such as color, shape, and textures because the object can be of any texture, color, shape or size. The objective of this research is to recognize image object using these features with high accuracy and low complexity and also to provide a comparative scenario among most used distance measures (as well as their ensemble) of finding nearest neighbors.

Many different researches on object recognition and its applications have been developed over past decades and are still undertaking plenty of research. In paper [1], authors propose an object detection and recognition model from videos. To classify the detected objects, they incorporate scale-invariant feature transform (SIFT) along with Tensor features using Deep Neural Network (DNN). Paper [2] discusses about the method of detecting mangoes from mango tree. To eradicate the dissimilar color or object in the image, they use color processing as key filtering, and use the edge detection and Circular Hough Transform (CHT) for shape revealing. The method determines the mango candidates and by collecting the maximum voting, they find the circular pattern with the given radius within a candidate image to automatically detect the target mango object and also count the total number of mangoes in it. For object detection problem, paper [3] introduces Context-Sensitive Decision Forests. To construct the trees, in combination with a priority 
based way they present an innovative split criterion. This technique permits more precise regression mode selection and therefore the method mends the current context information. They demonstrate improved results in comparison to upto-date methods for detecting pedestrian. In paper [4], authors compare five tree-based machine learning techniques based on random sub-windows framework: one Classification and Regression Tree (CART) based single-tree and four ensemble methods: Bagging, Boosting, CART based Random Forests, and Extra-Trees. They assess their system on three datasets where one dataset encompasses domestic objects in a precise environment (COIL-100) which reveals significant viewpoint variations, one dataset holds object classes in a precise environment (ETH-80) which reveals higher intra-class unevenness, and other dataset encompasses buildings in city scenes (ZuBuD) which comprises images with brightness, standpoint, scale and positioning variations as well as partial obstructions and messy backgrounds. They found decent outcomes when ensembles of decision trees are used, specifically when using Extra-Trees or Tree Boosting. The method of [5] is blend of two methods: Support Vector Machine (SVM) and K-Nearest Neighbor (KNN), where KNN is used to find nearby neighbors of the query object from all training image objects. Then they train a local SVM and used the trained model to recognize the query image object. For feature vector formation, Hu's translation, rotation and scaling invariant moments are figured to represent the image. They show their experimental results for COIL-100 database. Method [6] presents a classification system that can handle large known objects database with realtime performance using decision tree. Their method achieves 89 percent recognition rate for an image database of over 100,000 images which contains highly similar objects. Paper [7] presents a Boosting based generic object recognition framework which improves both the classification result and classifier stability. They also present a new segmentation technique. They obtained up to $87 \%$ classification results.

There are many research found but their direct results cannot be universal, though every approach has decent results within its own restricted situations. Henceforth there is effort to be done in blend of object detection technology in order to boost its performance. Again, one of the most significant challenges in object recognition is complexity of algorithm to make the system work in real time and for large dataset $[19,20]$. Most of the exiting works fail in standard of both high accuracy and low complexity at a time. In this paper, these cases are properly considered and solved to some extents. Moreover, we have outlined a comparative scenario among most used distance measures (as well as their ensemble) for object detection case. For this, first the images are resized and converted to gray scale image. To classify the object in image, low level or global features that means Color, Texture, and Shape features are used here. Hough transform, Gabor wavelet, and Color histogram are used to extract shape, texture, and colour features correspondingly. After Feature extraction, a vector of the features is formed for each image. To find the closest neighbor of the query image from the training images, distance measure is used. The class of that closest neighbor is considered as the class of the query image. To find the distance, we have used twelve different distance measures: Minkowski, City block or $\mathrm{L}_{1}$, Euclidean or $\mathrm{L}_{2}$, Chebyshev or $\mathrm{L}_{\max }$, Relative City block, Normalized $\mathrm{L}_{2}$, Cosine, Correlation, Relative deviation, Spearman, Canberra, and Standardized $\mathrm{L}_{2}$. Ensemble of these distance measures is also used. The recognition accuracies of the distance measures as well as their ensembles are compared. We assess the proposed system on COIL100 object-recognition dataset.

The rest of this paper is structured as follows. Detail materials and methods are clarified in Section 2. Experimental results are outlined in section 3 and the conclusion is sketched in section 4 .

\section{Materials and Methods}

The research comprises two major phases: training phase and testing phase. The holdout method is used for training and testing. Both phases contain some common steps such as image acquisition, preprocessing, feature extraction, and features vector creation. Rotation, Scaling, and Translation (RST) invariant features are used here in this method. Color histogram which is used here to extract color feature, concentrates merely on the quantity of the number of unalike sorts of colors, irrespective of the spatial locality of the colors [9]. Gabor wavelet which is used here to extract texture feature, lessens the ambiguity in information in both time and frequency domain [23]. Hough transform which is used here to extract shape feature, find deficient object instances by an elective process within a certain class of shapes [24]. RST invariant features help us to detect and recognize object when object in images has standpoint, orientation, positioning, and scale variations. The feature vectors are used to recognize the object by calculating the distance value between feature vector of each of the training image objects and query image object. The class of the training image object of minimum distance with query image object is considered as the predicted class label of the query image object. Algorithm 1 and algorithm 2 show the steps of the training and test phases respectively.

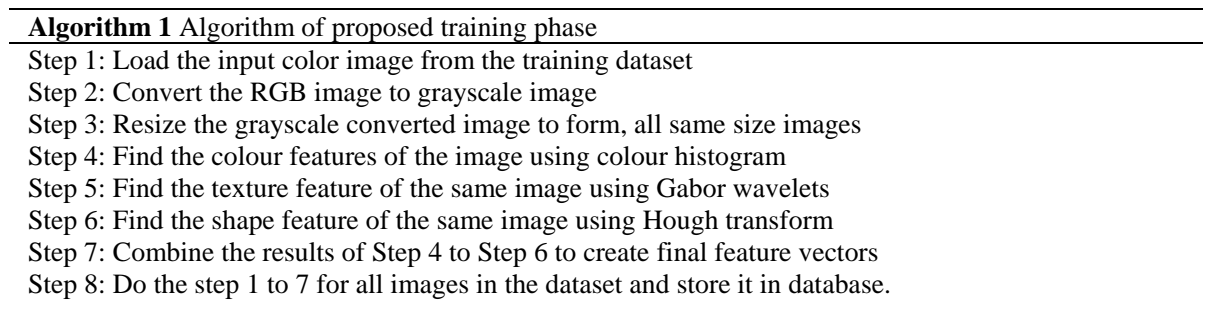




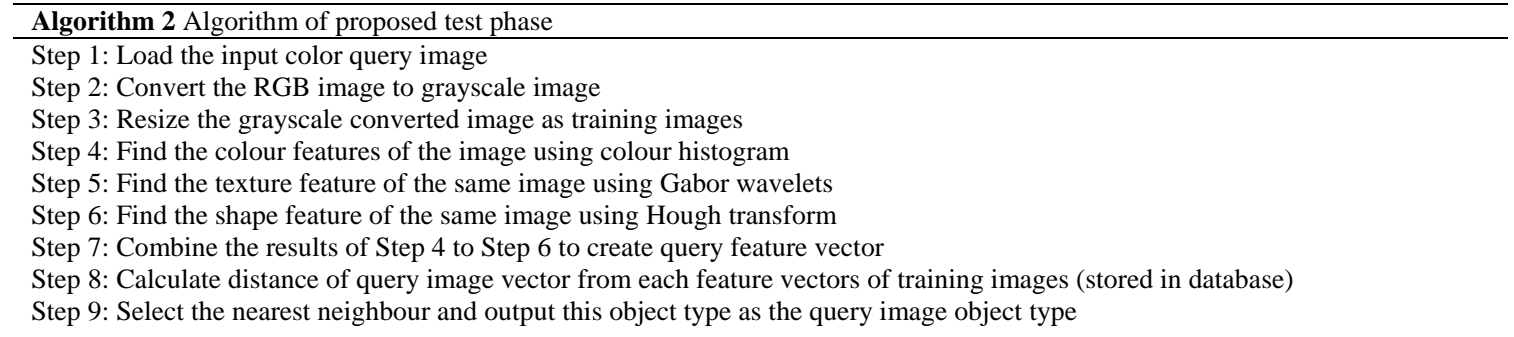

Fig.1 illustrates the proposed method architecture and the architecture is discussed in details below.

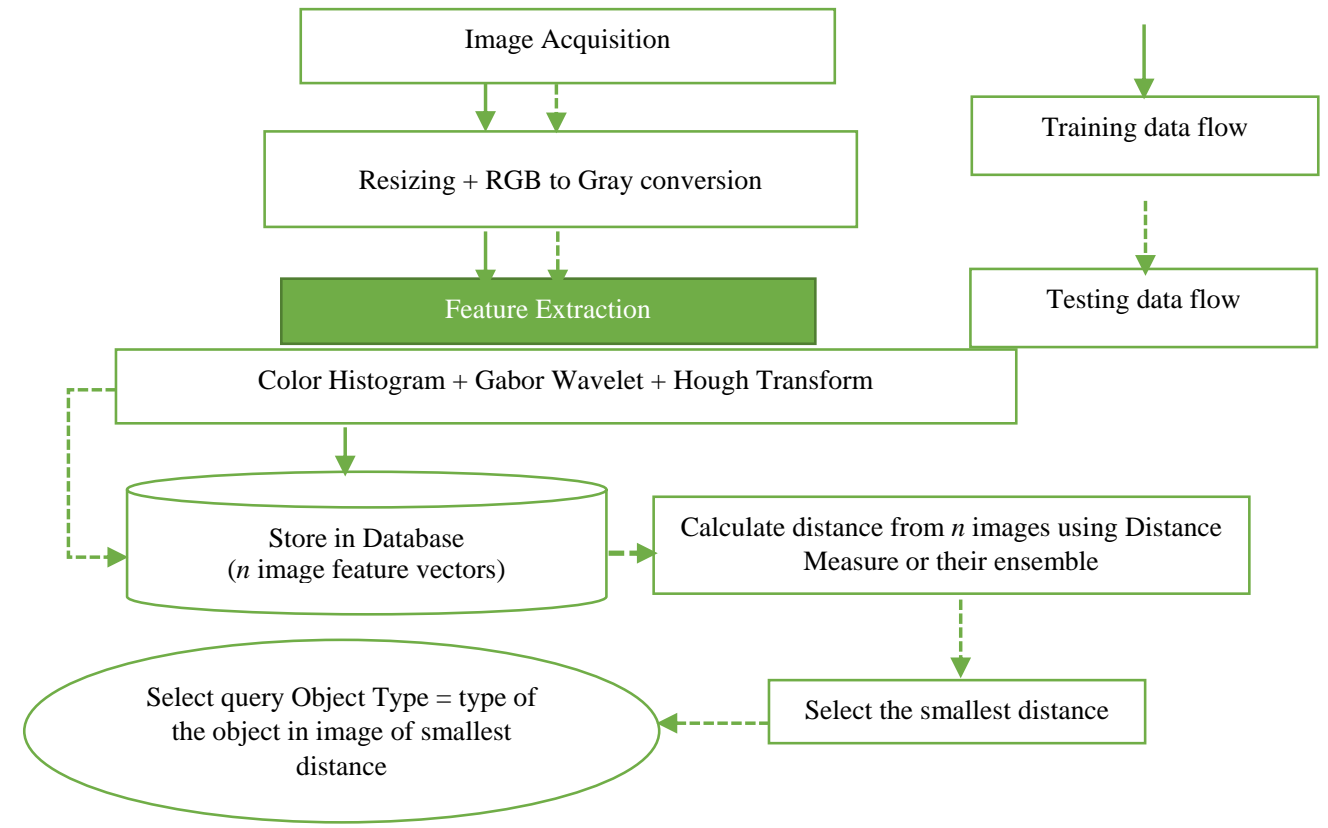

Fig. 1. Proposed Method Architecture.

\subsection{Image acquisition and preprocessing}

All images are resized to form same size images. Then the true colour images (image with an RGB colour map) are converted to the grayscale intensity images. We convert RGB to grayscale by removing the hue and saturation information while remembering the luminance. We transfigure RGB image to gray scale image because RGB is a device-dependent color model, even it differs in the same device over time [8]. Furthermore, gray scale image is one dimensional (whereas RGB image is 3 dimensional), so it reduces the computational complexity in feature extraction procedure. Conversion of RGB values to grayscale values, $I$ are performed by forming a weighted sum of the $R, G$, and $B$ channels using (1) [16].

$$
I=0.2989 * R+0.5870 * G+0.1140 * B
$$

\subsection{Feature extraction}

Objects are barely recognized by a machine unless it is trained to. The solution of training is to extract some information from object to detect and recognize it with the help of a resource having knowledge about that [20]. To extract information from the image, feature extraction is used. Features are extracted here using Color histogram, Gabor wavelet transform, and Hough transform of the converted grayscale image. A color histogram is a representation of the distribution of colors in an image [9]. The number of bins in the histogram of gray image is 256. Each bin in the color histogram represents the frequency or number of pixels of corresponding color. These frequencies of colors are used as color histogram features i.e. we get total 256 color features.

Gabor wavelet diminishes the product of its standard deviations i.e. it minimizes the uncertainty in information carried by this wavelet both in the time and frequency domain. But the limitation is that this wavelet is non-orthogonal, so it is problematic for proficient decomposition into the origin [10][11]. We calculate Gabor features of the grayscale image using (2), which is Gaussian restrained by a complex exponential [12].

$$
f(x)=e^{-\left(x-x_{0}\right)^{2} / a^{2}} e^{-i k_{0}\left(x-x_{0}\right)}
$$


Here, $x_{0}$ represents the distance from the center, the rate of the exponential drop-off is controlled by $a$ and the rate of modulation is controlled by $k_{0}$. For each scale and orientation, we calculate mean-squared energy and mean amplitude. To do this, we calculate the median-squared energy. Median is calculated over orientations which means for a particular scale, orientation is varied to take the median [13] and from this, we estimate the mean. Instead of normal addition, our measure of phase symmetry is average through all scales and orientations. Thus, we get total 30 Gabor wavelet texture features for each image.

To extract shape features, first we detect edges using the canny method which finds edges by beholding for local maxima of the gradient of image (in our case, grayscale image). We use two thresholds to detect strong and weak edges, even those weak edges in the output if they are connected to strong edges. Because of using two thresholds, this method is less noise sensitive than other methods and can detect more true weak edges [14].

Then, we detect lines using Standard Hough Transform (SHT), H, of the binary image (extracted canny edge). Standard Hough Transform is a parameter space matrix whose rows and columns correspond to rho (the distance from the origin to the line along a vector perpendicular to the line) and theta (the angle in degrees between the $x$-axis and this vector) values respectively [15]. After that, we calculate one mean value, one standard deviation value, and one Skewness values of the elements in $H$. Thus we get total 3 Hough transform based shape features.

\subsection{Feature vector creation and storing in database}

A feature vector is created using the extracted 289 features (256 color features, 30 texture features, and 3 shape features) for each image. Then the feature vectors are warehoused in the store.

\subsection{Object classification using distance measures}

To make recognition result very apposite, classification analyses the extracted features. In testing phase, the features of the query image are mined and vector of these features is created using the same steps as described before. To classify the object in query image, we use the nearest neighbor. To do this, one of the distance measures is used to find the distances between the query image feature vector and feature vectors of each of the training images (which are warehoused in database). The class of image in the database which has maximum similarity (i.e. nearest neighbor) is considered as the class of the object of the query image. The distance measures used here for finding similarity are: Minkowski, City block or $\mathrm{L}_{1}$, Euclidean or $\mathrm{L}_{2}$, Chebyshev or $\mathrm{L}_{\max }$, Relative City block, Normalized $\mathrm{L}_{2}$, Cosine, Correlation, Relative deviation, Spearman, Canberra, and Standardized $\mathrm{L}_{2}$. Moreover, the ensemble of the mentioned distance measures is also used. Here in ensemble, each distance measure predicts the class of the query object and the final class is selected based on majority.

\section{Experimental Results}

We implement our methods in MATLAB R2016b platform. At the beginning, we start to get a dataset of images. A number of crowdsourcing datasets are available for research. To evaluate our method, we have used COIL-100 dataset.

\subsection{Dataset Description}

The COIL-100 dataset comprises household objects in a defined environment which exposes significant viewpoint differences. The dataset contains total 7200 images of 100 different types of objects. That means, for each type of object there have 72 images of different degrees of object ( 0 degree to 355 degree, each 5 degree apart). For testing, we have used images of 90 and 180 degree rotated objects. Images of object of other degrees are used for training i.e. from each object type we have used 2 images for testing and 70 images for training. So finally, the training set contains 7000 images and the test set contains 200 images. Fig. 2 shows sample training images of an object in various angles and fig. 3 shows some query images.
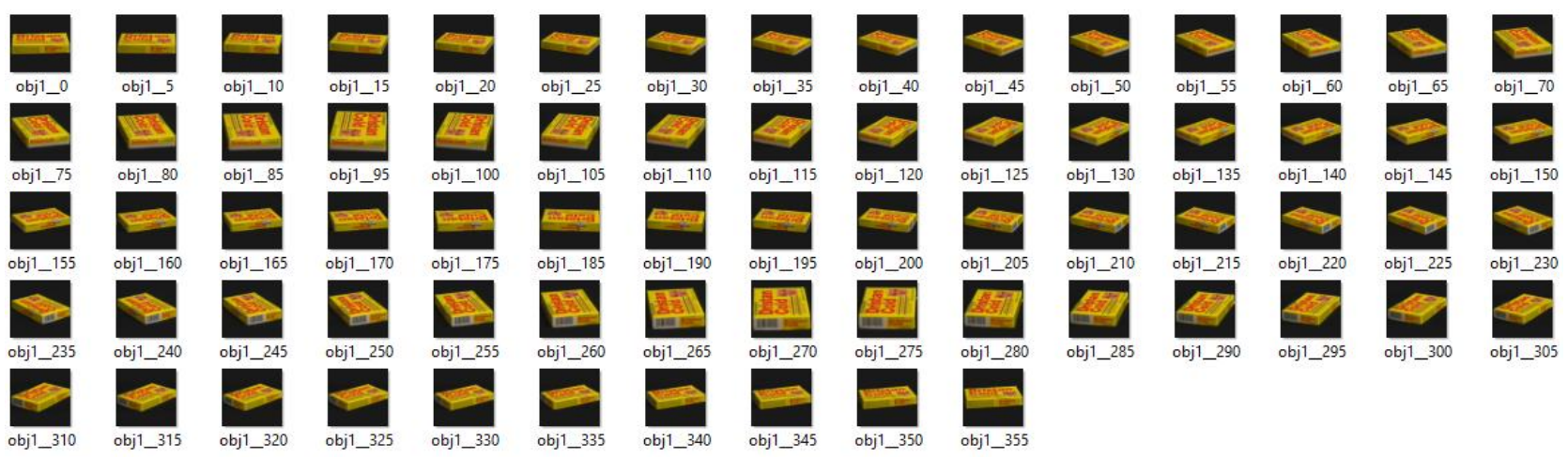

Fig. 2. A sample training image object in various angles 


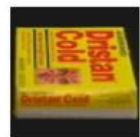

obj1_90

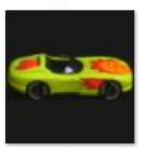

obj6_180

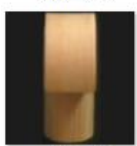

obj12_90

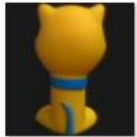

obj17_180

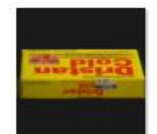

obj1_180

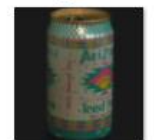

obj7 90

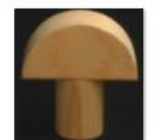

obj12_180

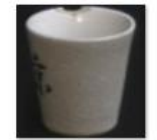

obj18_90
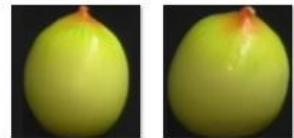

obj2_180
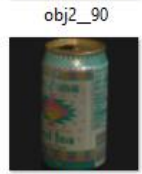

obj7_180

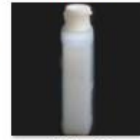

obj13_90

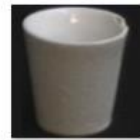

obj18_180

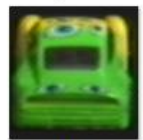

obj8_90

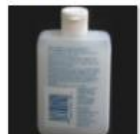

obj13_180

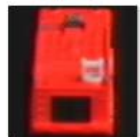

obj19_90

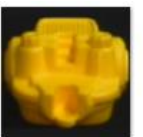

obj3_90

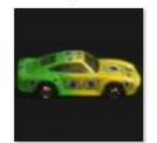

obj8_180

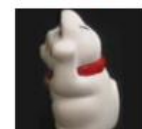

obj14_90

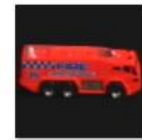

obj19_180

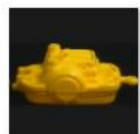

obj3_180

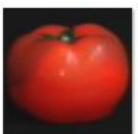

obj4_90

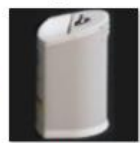

obj9_90

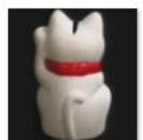

obj14_180

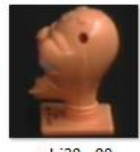

obj20_90

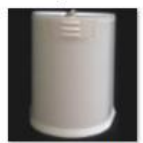

obj9_180

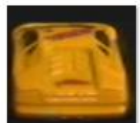

obj15_90

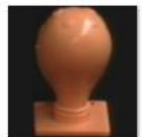

obj20_180

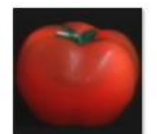

obj4_180

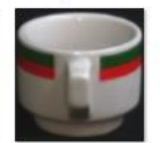

obj10_90

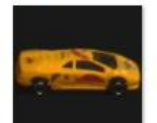

obj15_180

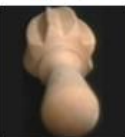

obj21_90

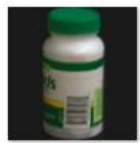

obj5_90

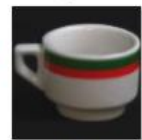

obj10_180

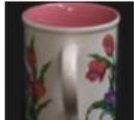

obj16_90

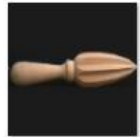

obj21_180

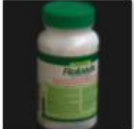

obj5_180

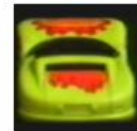

obj6_90

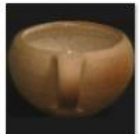

obj11_90

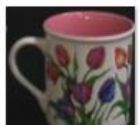

obj16_180

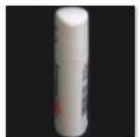

obj22_90

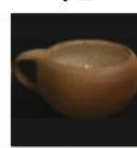

obj11_180

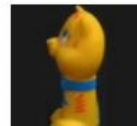

obj17_90

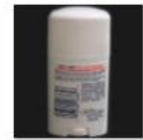

obj22_180

Fig. 3. Sample query image objects in two different angles

\subsection{Experiment}

First, all images are resized to $400 \times 300$. When extracting histogram based features, the input is the gray scale converted images and the output we get is 256 color features. Fig. 4(a) and 4 (b) show a sample RGB image of the dataset and its gray level converted image respectively, 256 bins in the histogram of this image are shown by $\mathrm{x}$-axis in fig. 4(c) and $y$-axis shows the number of pixels of the corresponding bin.

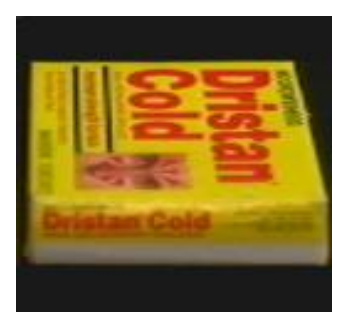

(a)

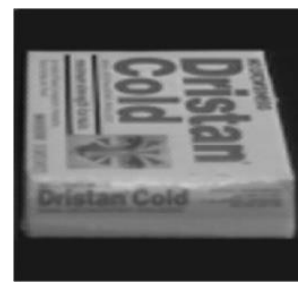

(b)

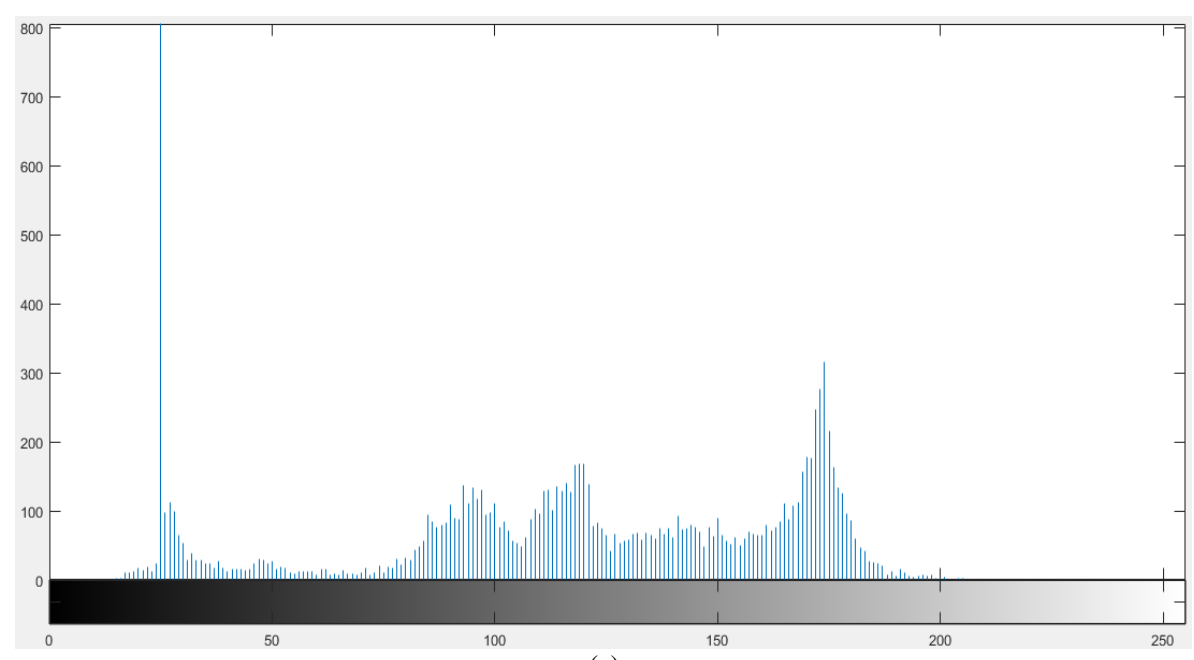

(c)

Fig. 4. (a) One sample RGB image of the dataset (b) Gray level image (c) colour histogram of the image in (b)

When extracting Gabor wavelet features, the input is the gray scale converted images and the output we get is thirty texture features. To get this, we use default number of wavelet scales $=5$, Number of filter orientations $=6$, Wavelength of smallest scale filter $=3$, Scaling factor between successive filters $=2.1$, Ratio of the standard deviation of the Gaussian to the filter centre frequency $=0.55$, Ratio of angular interval between filter orientations and the standard deviation of the angular Gaussian function $=1.2$, No of standard deviations of the noise energy beyond the mean $=2$. Moreover, we use polarity $=0$, i.e. we look for both black and white spots of symmetry. We extract the Gabor wavelet based texture feature from time domain of the image. Fig. 5 shows the 30 Gabor wavelet features for sample image in fig. 4(b). 


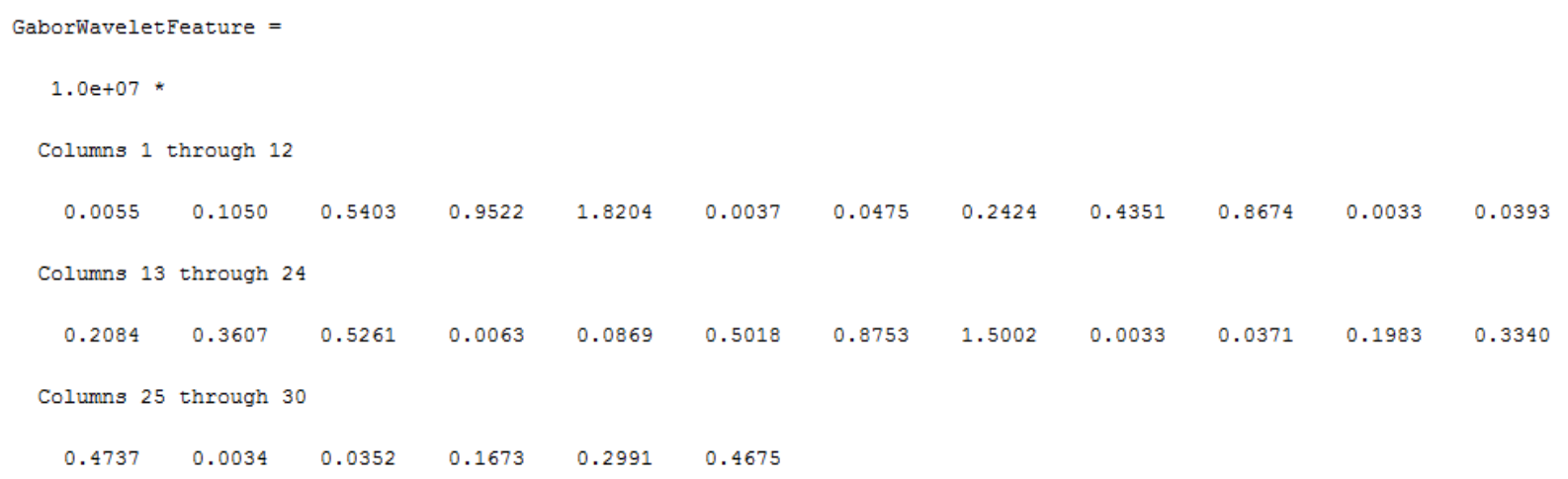

Fig. 5. Thirty Gabor Wavelet Features for image in fig. 4(b)

When extracting Hough features, the input is the gray scale converted images and the output we get is, three shape features. To get this, we first extract the canny edge of the grayscale images and for this, the high threshold value is chosen randomly and 0.4 multiplied high threshold value is used for the low threshold. Fig. 6(a) shows the binary canny edge image of sample image 4(a) of our dataset. To detect lines using SHT from Binary canny edge image, we use 0.5 spacing of Hough transform bins along the rho axis and we use theta value within the range [-90, 0.5, 89] for the corresponding column of the Hough transformed image. Fig. 6(b) shows a portion (67\%) of Hough Transformed image of fig. 6(a). Then, we calculate the first three moments from Hough transformed matrix. As an example, we get mean = 3.7291, standard deviation $=6.3938$, and Skewness $=1.7557$ from fig. 6(b) for sample image of 4(a).

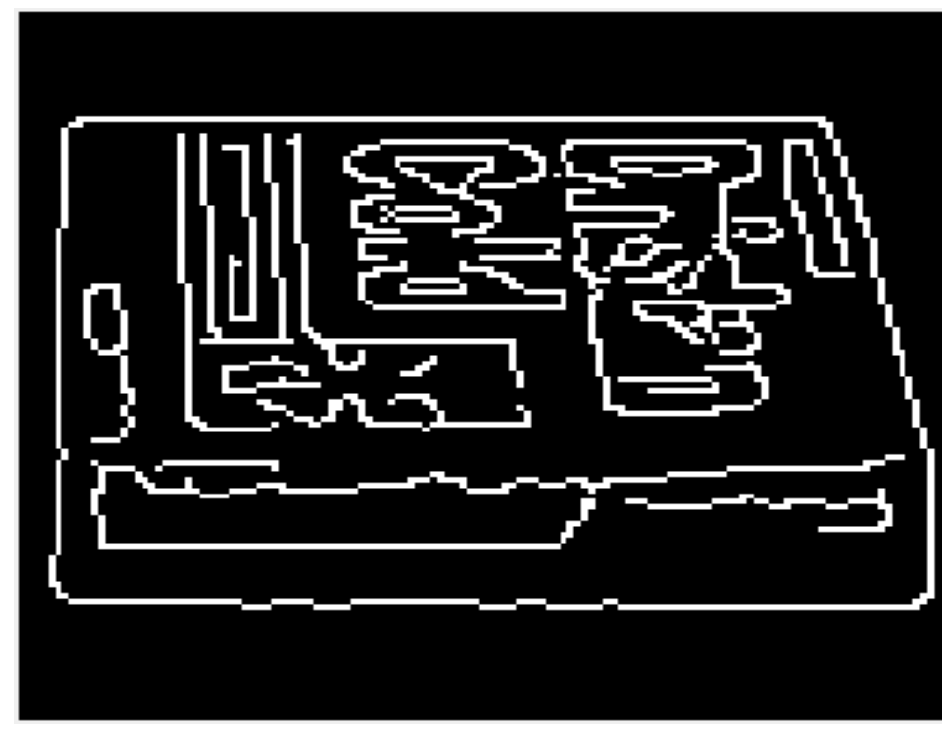

(a)

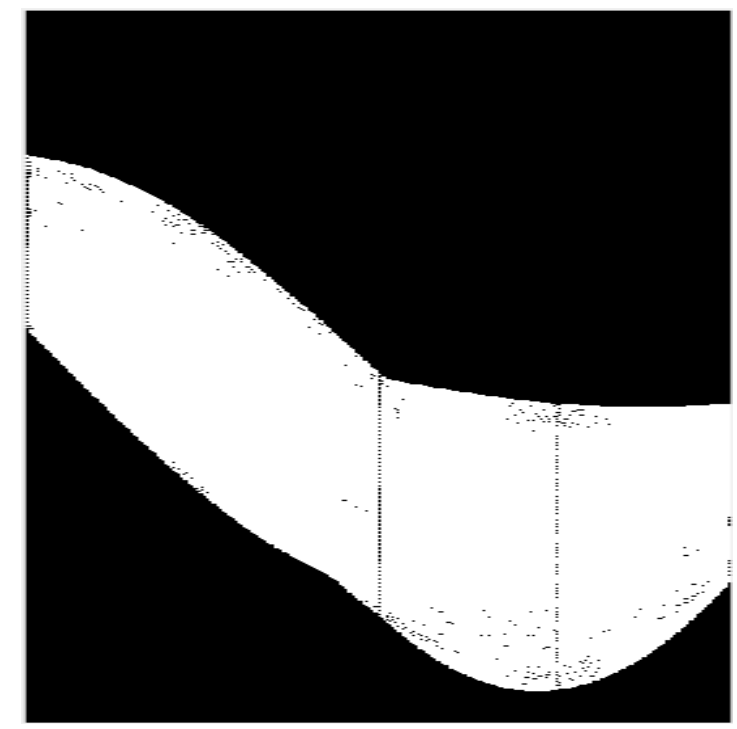

(b)

Fig. 6. (a) Canny edge of object of image in fig. 4(b) (b) 67\% Portion of Standard Hough Transform of Binary Canny edge image of (a)

\subsection{Evaluation}

Among 200 query image objects, 157 query objects are correctly recognized by all of the distance measures used in this method and their ensemble. Among other 43 query objects, some distance measures (and/or ensemble of all 12 distance measures) recognize them correctly shown by blank space and some distance measures (and/or their ensemble) recognized them incorrectly shown by red color in fig. 7. That means fig. 7 shows the screenshot of error portion of our output (matrix of those 43 objects), where object number in black color shows the original object. In the following figure, object name such as obj51_275 indicates 275 degree angled 51 number object of the dataset. 


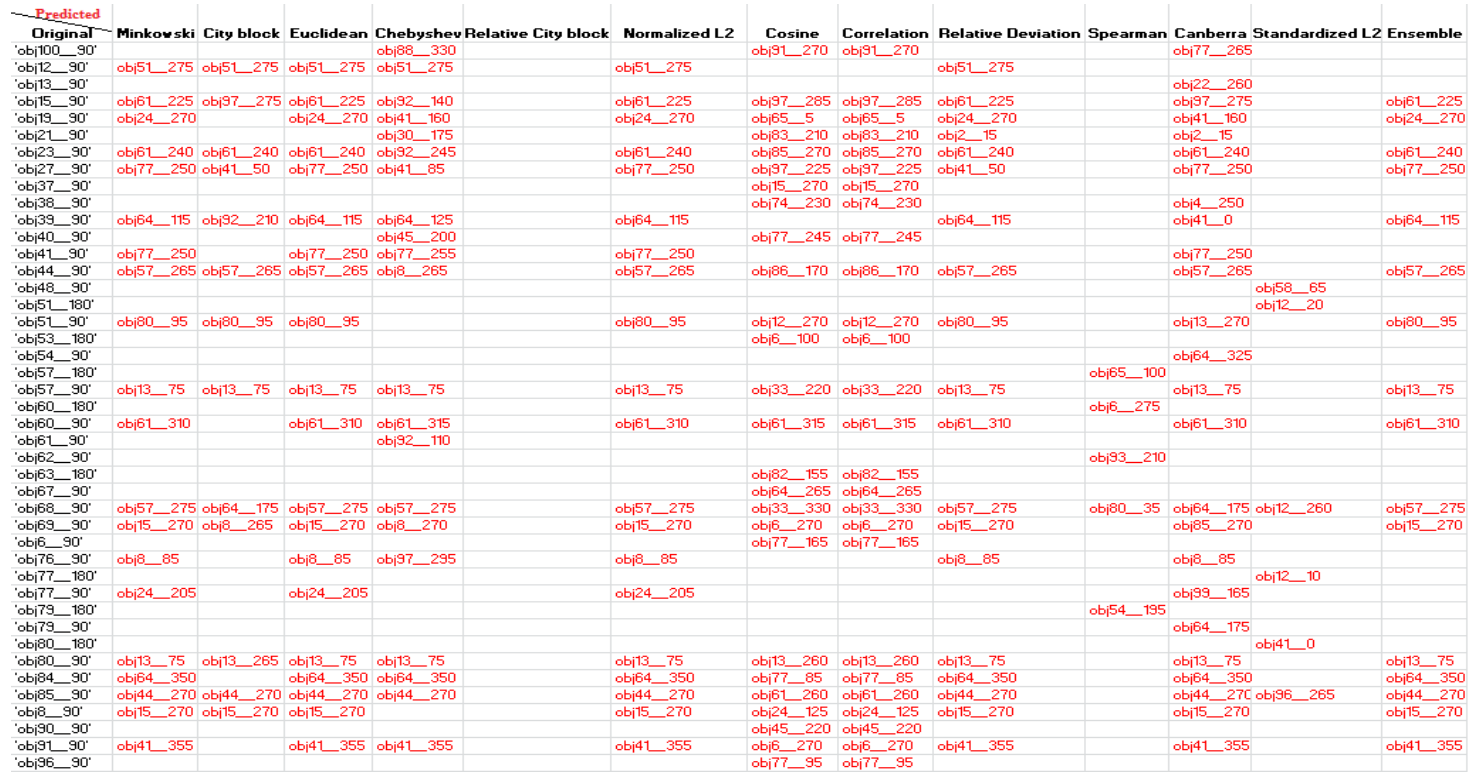

Fig. 7. Error output Matrix

The $2^{\text {nd }}$ column of table 1 shows this total number of incorrectly predicted objects by various distance measures and their ensemble and the accuracy of object classification using 12 different distance measures and their ensemble for COIL-100 dataset is shown in $3^{\text {rd }}$ column of the table below (Final row of the table shows the result for ensemble of 12 mentioned distance measures).

Table 1. Result for 200 query image objects of COIL-100 dataset

\begin{tabular}{|c|c|c|}
\hline Distance & Incorrectly Predicted & Accuracy \\
\hline Minkowski & 20 & $90 \%$ \\
\hline City block & 13 & $93.50 \%$ \\
\hline Euclidian & 20 & $90 \%$ \\
\hline Chebyshev & 21 & $89.50 \%$ \\
\hline Relative City block & 0 & $100 \%$ \\
\hline Normalized L2 & 20 & $90 \%$ \\
\hline Cosine & 26 & $87 \%$ \\
\hline Correlation & 26 & $87 \%$ \\
\hline Relative Deviation & 19 & $90.50 \%$ \\
\hline Spearman & 5 & $97.50 \%$ \\
\hline Canberra & 25 & $87.50 \%$ \\
\hline Standardized L2 & 6 & $97 \%$ \\
\hline Ensemble of 12 distances & 16 & $92 \%$ \\
\hline
\end{tabular}

Fig. 8 and fig. 9 show the comparative miss-prediction and performance respectively, for 12 distance measures (used in this method) and their ensemble.

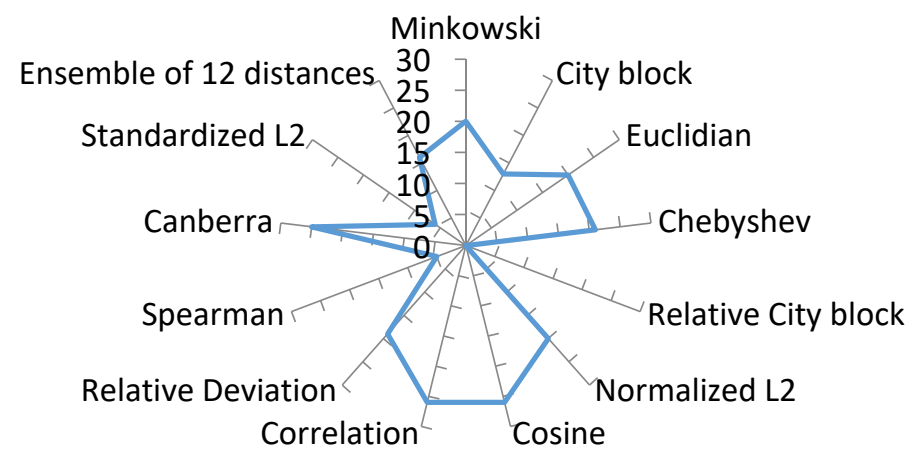

Fig. 8. Miss-prediction comparison among distance measures and their ensemble 


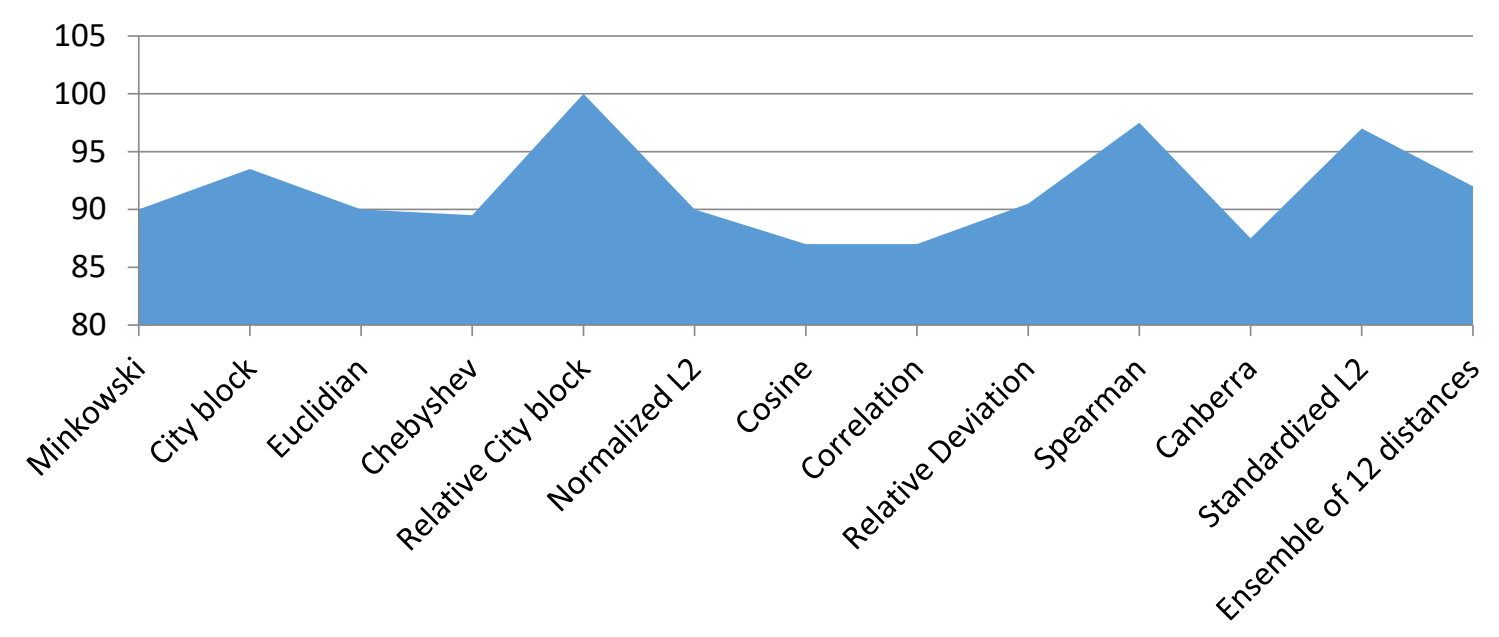

Fig. 9. Performance comparison among distance measures and their ensemble

Experimental results show that for finding appropriate nearest neighbor, cosine and correlation measures are less effective than other measures used here which show the worst accuracy ( 87 percent for both measures), whereas the Relative city block is the robust distance measure here for object classification because it calculates the absolute distance differences. Besides Relative city block, Spearman and standardized Euclidean measures also show good competitive results. The ensemble of the distance measures shows 92 percent accuracy, thus ensemble of distance measures is not a better choice in this case.

\section{Conclusion}

In this research, three global features: color, texture, and shape features are used to recognize unknown object in image. To extract these features (color, surface structure, and contour), color histogram, Gabor wavelet, and Hough transform are used respectively. These global features are used for feature vector creation of each image. For recognition, nearest neighbor is used i.e. object with shortest distance or maximum similarity of the training image is recognized as the query image object. Twelve different distance measures are used here and ensemble of these twelve distance measures is also used for object recognition in the image. The research is evaluated on a publicly available object-recognition dataset. The stated results ratify that the recognition accuracy using relative city block outperforms many up-to-date methods. There are various existing methods that only represent the high accuracy or low complexity, but don't focus on both high accuracy and low complexity at a time. So these researches are not suitable to face the challenges of large dataset. One of the major contributions of this paper is that this work advances the field from the present state of knowledge in terms of high accuracy with low complexity because the distance measure is mathematically sound and also easy to implement. Again, so far we know, no related work exists that shows comparative analysis among all most used distance measures and their ensemble for object classification.

\section{References}

[1] Najva, N., K. Edet Bijoy. SIFT and tensor based object detection and classification in videos using deep neural networks. Procedia Computer Science 2016; 93: 351-358.

[2] Hussin, R., Juhari, M. R., Kang, N. W., Ismail, R. C., Kamarudin, A. Digital image processing techniques for object detection from complex background image. Procedia Engineering 2012; 41: 340-344.

[3] Peter, K., Rota Bulò, S., Antonio, C., Pushmeet, K., Marcello, P., Horst, B. Context-Sensitive Decision Forests for Object Detection 2012; 440-448.

[4] Marée, R., Geurts, P., Piater, J., Wehenkel, L. Decision trees and random subwindows for object recognition. In ICML workshop on Machine Learning Techniques for Processing Multimedia Content (MLMM2005), 2005.

[5] Muralidharan, R., Chandrasekar, C. Object recognition using SVM-KNN based on geometric moment invariant. International Journal of Computer Trends and Technology. 2011 Aug; 1(1):215-20.

[6] Blackwell, P., Austin, D. Appearance Based Object Recognition with a Large Dataset using Decision Trees. In Proceedings of the Australasian Conference on Robotics and Automation, 2004.

[7] Opelt, A., Pinz, A., Fussenegger, M., Auer, P. Generic object recognition with boosting. IEEE Transactions on Pattern Analysis and Machine Intelligence. 2006 Jan 23; 28(3):416-31.

[8] RGB color model. https://en.wikipedia.org/wiki/RGB_color_model. Last accessed date: 23 March, 2021.

[9] Color histogram. https://en.wikipedia.org/wiki/Color_histogram. Last accessed date: 23 March, 2021.

[10] Lee TS. Image representation using 2D Gabor wavelets. IEEE Transactions on pattern analysis and machine intelligence. 1996 Oct;18(10):959-71. 
[11] Daugman, John. Computer Vision Lecture Series. University of Cambridge.

[12] Daugman, John. Information Theory Lecture Series. University of Cambridge.

[13] Kuse, Manohar, Yi-Fang Wang, Vinay Kalasannavar, Michael Khan, and Nasir Rajpoot. "Local isotropic phase symmetry measure for detection of beta cells and lymphocytes." Journal of Pathology Informatics 2, 2011.

[14] Edge. https://www.mathworks.com/help/images/ref/edge.html. Accessed date: 30 March, 2021.

[15] Hough. https://www.mathworks.com/help/images/ref/hough.html. Accessed date: 30 March, 2021.

[16] Rgb2gray. https://www.mathworks.com/help/matlab/ref/rgb2gray.html. Accessed date: 30 March, 2021.

[17] Cai, Fei, Honghui Chen, and Jianwei Ma. "Man-made object detection based on texture visual perception." International Journal of Engineering and Manufacturing (IJEM), MECS, Vol. 2, no. 3, pp. 1-8, 2012.

[18] Mani, M. Radhika, G. P. S. Varma, D. M. Potukuchi, and Ch Satyanarayana. "Design of a novel shape signature by farthest point angle for object recognition." I.J. Image, Graphics and Signal Processing (IJIGSP), MECS, Vol. 7, no. 1, pp. 35-46, 2015.

[19] Mishra, P.K. and Saroha, G.P., A study on classification for static and moving object in video surveillance system. International Journal of Image, Graphics and Signal Processing (IJIGSP), MECS, Vol. 8, No. 5, pp.76-82, 2016.

[20] Jain, S.K. and Rajankar, S.O., Real-Time Object Detection and Recognition Using Internet of Things Paradigm. International Journal of Image, Graphics and Signal Processing, (IJIGSP), MECS, Vol. 9, No. 1, pp.18-26, 2017.

[21] Khalifa, F.A., Semary, N.A., El-Sayed, H.M. and Hadhoud, M.M.. Local detectors and descriptors for object class recognition. International Journal of Intelligent Systems and Applications (IJISA), MECS, Vol. 7, No. 10, pp.12-18, 2015.

[22] Ma, L., Liu, Y., Jiang, H., Wang, Z. and Zhou, H. An improved method of geometric hashing pattern recognition. International Journal of Modern Education and Computer Science (IJMECS), MECS, Vol. 3, No. 3, pp.1-7, 2011.

[23] https://en.wikipedia.org/wiki/Gabor_wavelet. Accessed date: 31 March, 2021.

[24] https://en.wikipedia.org/wiki/Hough_transform. Accessed date: 31 March, 2021.

\section{Authors' Profiles}

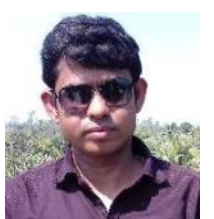

S.M. Mohidul Islam received his Bachelor of Science in Computer Science and Engineering and Master of Science in Computer Science and Engineering degrees from Science, Engineering and Technology School, Khulna University, Bangladesh. He is currently working as a faculty member in Computer Science and Engineering Discipline, Khulna University. His research interest includes Data Science and Machine Learning for Community ICT (ICT solutions for real life problems/healthiness of terminal people).

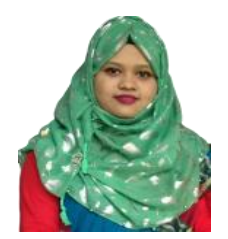

Farhana Tazmim Pinki received her Bachelor of Science in Computer Science and Engineering and Master of Science in Computer Science and Engineering degrees from Science, Engineering and Technology School, Khulna University, Bangladesh. She is currently working as a faculty member in Computer Science and Engineering Discipline, Khulna University. Her research interest includes Machine Learning, Data Mining, and Digital Image Processing.

How to cite this paper: S.M. Mohidul Islam, Farhana Tazmim Pinki, " Colour, Texture, and Shape Features based Object Recognition Using Distance Measures", International Journal of Engineering and Manufacturing (IJEM), Vol.11, No.4, pp. 42-50, 2021. DOI: $10.5815 / \mathrm{ijem} .2021 .04 .05$ 